Department of Social Systems and Management

$$
\text { Discussion Paper Series }
$$

\author{
No. 1173 \\ Impact of Skeleton Imports on Hollowing Out \\ Production Bases Outside Japan
}

by

Ushio Sumita and Rina Isogai

June 2007

UNIVERSITY OF TSUKUBA

Tsukuba, Ibaraki 305-8573

JAPAN 


\title{
Impact of Skeleton Imports on Hollowing Out Production Bases Outside Japan
}

\author{
Ushio Sumita ${ }^{1}$, Rina Isogai ${ }^{2}$ \\ ${ }^{1}$ Graduate School of Systems and Information Engineering, University of Tsukuba, Tsukuba, Japan \\ ${ }^{2}$ Graduate School of Systems and Information Engineering, University of Tsukuba, Tsukuba, Japan
}

\begin{abstract}
Many Japanese manufacturing companies have moved their production facilities partially or completely to China and other Asian countries, and the products produced at such oversea production bases are reversely imported into Japan. While this hollowing out production bases outside Japan enables them to reduce the labor and other operation costs, it incurs the extra logistics costs. In addition, the market lead time becomes inevitably longer, resulting in the increased inventory costs. In order to compensate these negative effects, certain Japanese companies have been implementing the SI (Skeleton Imports) strategy, where a variety of products in small quantity would be designed in such a way that a common frame (called Skeleton) can be used for all the products and various product specifications can be mounted onto the common frame to produce a variety of products in small quantity efficiently. The purpose of this paper is to analyze the positive effects of the SI strategy via simulation.
\end{abstract}

Keywords - Hollowing out, logistics losses, skeleton imports

\section{INTRODUCTION}

During the past decade, the Internet has impacted the way businesses are conducted across all industries. In manufacturing of consumer products, because of the Internet, the industry has been shifting rapidly from "production based on demand estimates" to "production based on confirmed orders." In light of a variety of products in small quantity demanded by the consumer market, this change enables one to reduce the inventory costs significantly, and is now one of the key factors to be competitive in manufacturing.

In order to facilitate the order-based production, an efficient information infrastructure is absolutely necessary, synchronizing all business activities throughout the value chain from sales and services in the downstream to procurement of parts and materials in the upstream. The necessary information infrastructure has become readily available by creating an intranet on the Internet. In printer business, for example, the confirmed orders of a day would be sent to a manufacturing plant from the sales offices across Japan through the company's intranet. During the night, the production plan of the next day would be established based on these confirmed orders. A typical production lead time for printers is one day, and the ordered products would be produced by the end of the next day, and packaged and shipped out on the following day. Accordingly, the market lead time from the generation of an order to the delivery of the products would be within three days.

Many Japanese manufacturing companies have moved their production facilities partially or completely to China and other Asian countries. In the literature, many different factors are discussed for motivating outward FDI (Foreign Direct Investment), including heterogeneity in productivity among domestic firms [1] [2], networks to sell products to buyers from the same country [3], rapid demand growth in the FDI host country [4], and cheaper costs for labor and others [5] [6]. For the case of Japan, the last two factors seem to be most important. Other references concerning FDI and the hollowing out effects include [7], [8] and [9].

While moving the production facilities abroad may reduce the production costs significantly, it also inevitably incurs additional logistics costs at the same time. Furthermore, the market lead time would be prolonged and the order-based production becomes impossible because of the transportation time over several days. In order to compensate these negative effects, certain Japanese companies have been implementing the SI (Skeleton Imports) strategy, where a variety of products in small quantity would be designed in such a way that a common frame (called Skeleton) can be used for all the products and various product specifications can be mounted onto the common frame to produce a variety of products in small quantity efficiently. While Skeletons are produced abroad and imported to Japan, manufacturing operations to meet a variety of product specifications are done at logistics centers in Japan. Such a combination of production of Skeletons abroad and value-added logistics within Japan enables one to take advantage of cost reduction by producing abroad and to maintain the almost-order-based production at the same time. The purpose of this paper is to examine the impact of this new phenomenon through Monte Carlo simulation.

\section{MODEL DESCRIPTION}

In this section, a production system is considered where one type of products with $K$ different variations would be produced. These variations are written as product $i, i=1,2, \cdots, K$. With time unit taken to be a day, the demand of product $i$ during day $t$ is denoted by $D_{i}(t)$, $t=1,2, \cdots, T$. Three different models are introduced in order to evaluate the effects of the SI strategy for compensating 
logistics loses and other negative effects caused by moving production bases outside Japan.

\section{A. Model I: Domestic Production}

Model I corresponds to a case where the products are produced based on confirmed orders at a centralized manufacturing plant within Japan, where the production lead time of any product is assumed to be one day. Let $X_{I: i}(t)$ be the production quantity of product $i$ during day $t$. Because of the order-based production with the production lead time of one day, one has

$$
X_{t i}(t+1)=D_{i}(t)
$$

A set of parts necessary for producing one unit of product $i$, however, would require the procurement lead time of $k_{I: i}$ days. In other words, an order placed at the beginning of day $t-k_{I: i}+1$ would be delivered to the manufacturing plant (M.P.) at the end of day $t$. Assuming that the safety stock is set to cover the production of next $m_{I}$ days, one has to estimate the demands until day $t+m_{I}$ in order to determine the order quantity at the beginning of day $t-k_{I: i}+1$. More specifically, let $\hat{D}_{i}(\tau, t)$ and $\hat{X}_{I: i}(\tau, t)$ be the estimates of $D_{i}(t)$ and $X_{I: i}(t)$ respectively estimated at the beginning of day $\tau$, and define $Q_{I: i}(t)$ to be the order quantity of the parts for product $i$ placed at the beginning of day $t$. Furthermore, let $I_{I: i}(t)$ be the inventory level of product $i$ at the end of day $t$. One then sees that

$$
\begin{gathered}
Q_{I: i}\left(t-k_{I: i}+1\right)=\hat{D}_{i}\left(t-k_{I: i}+1, t+m_{I}-1\right) \\
=\hat{X}_{I: i}\left(t-k_{I: i}+1, t+m_{I}\right)
\end{gathered}
$$

and

$$
I_{I: i}(t)=\left[\tilde{I}_{I: i}(t)\right]^{+} ; \tilde{I}_{I: i}(t)=I_{I: i}(t-1)+Q_{I: i}\left(t-k_{I: i}+1\right)-X_{I: i}(t),
$$

where $[x]^{+}=\max \{x, 0\}$. Let $f_{I}\left(T_{1}, T_{2}\right)$ be the overall profit generated from the products delivered to the market between day $T_{1}$ and day $T_{2}$. Assuming that the delivery lead time is one day, one has

$$
\begin{aligned}
f_{I}\left(T_{1}, T_{2}\right) & =\sum_{\tau=T_{1}-2}^{T_{2}-2} \sum_{i=1}^{K}\left\{D_{i}(\tau-1)+\delta\left\{\tilde{I}_{I: i}(\tau)<0\right\} \cdot \tilde{I}_{I: i}(\tau)\right\} \cdot\left(P_{i}-T C_{i}-c_{I: i}-O P_{I: i}\right) \\
& -\sum_{\tau=T_{1}-1}^{T_{2}-2} \Sigma_{i=1}^{K} \frac{I_{I: i}(\tau-1)+I_{I: i}(\tau)}{2} \cdot c_{I: i} \cdot r \\
& +\sum_{\tau=T_{1}-2}^{T_{2}-2} \sum_{i=1}^{K} \delta\left\{\tilde{I}_{I: i}(\tau)<0\right\} \cdot \tilde{I}_{I: i}(\tau) \cdot C L_{i}
\end{aligned}
$$

where $\delta\{P\}=1$ if statement $P$ holds true, and $\delta\{P\}=0$ otherwise. Here, $P_{i}$ and $T C_{i}$ are the price and the transportation cost respectively of one unit of product $i$, $c_{I: i}$ and $O P_{I: i}$ are the parts cost and the operation cost respectively for producing one unit of product $i$, and $r$ is the inventory holding cost. In addition, $C L_{i}$ is the cost per unit of product $i$ for losing confidence by failing to meet the demand.

\section{B. Model II: Hollowing-Out Production Bases}

In Model II, the oversea M.P. would produce all the final products, which would be received at the distribution center (D.C.) inside Japan and then delivered to the market. It is assumed that the transportation lead time between M.P. and D.C. would be $l$ days. Despite this transportation delay, the supply chain is managed in such a way that the market lead time is reduced from three days to two days, enhancing the service in the eyes of the customers at the expense of the increased inventory cost. In other words, the confirmed orders of day $t-1$ would be sent to D.C. from the sales offices across Japan through the company's intranet. During day $t$, the ordered products would be packaged and shipped out, resulting in the market lead time of two days, provided that the inventory stock is large enough.

At D.C., the safety stock of the final products would be to cover $m_{I I: D . C \text {. }}$ days worth of the estimated demands. Let $S P_{I I: D . C: i}(t)$ be the quantity of product $i$ to be shipped from M.P. to D.C. at the beginning of day $t$. The inventory level of product $i$ at D.C. at the end of day $t$ is denoted by $I_{I I: D . C: i}(t)$. We also define $X_{I I: i}(t)$ to be the production quantity during day $t$ at M.P. One then sees that

$$
S P_{I I: D . C: i}(t-l+1)=X_{I I: i}(t-l)=\hat{D}_{i}\left(t-l, t+m_{I I: D . C .}-1\right),
$$

and

$$
\begin{aligned}
& I_{I I: D . C: i}(t)=\left[\widetilde{I}_{I I: D . C: i}(t)\right]^{+} ; \\
& \widetilde{I}_{I I: D . C: i}(t)=I_{I I: D . C: i}(t-1)+S P_{I I: D . C: i}(t-l+1)-D_{i}(t-1) .
\end{aligned}
$$

Here, the products shipped from M.P. to D.C. at the beginning of day $t-l+1$ would be produced at M.P. during day $t-l$. This delivered quantity, to reach D.C. at the end of day $t$, would be to cover the processing at D.C. for day $t+m_{I I: D . C \text {. }}-1$, explaining (5). Equation (6) states that the inventory level at the end of day $t$ would be the sum of the inventory level at the end of day $t-1$ and the delivered quantity at the end of day $t$ minus the demand of day $t-1$ processed during day $t$.

At M.P., the procurement lead time is assumed to be $k_{I I: i}$ days, and the safety stock for the parts would be to cover $m_{I I: M . P .}$ days worth of manufacturing operation at M.P. Let $Q_{I I: M . P . i}(t)$ and $\hat{X}_{I I: i}(\tau, t)$ be defined similarly to $Q_{I: i}(t)$ and $\hat{X}_{I: i}(\tau, t)$ respectively. As for Model I, one then sees that

$$
\hat{X}_{I I: i}(\tau, t)=\hat{D}_{i}\left(\tau, t+l+m_{I I: D . C .}-1\right) .
$$


Accordingly, it follows that

$$
\begin{aligned}
Q_{I I: M . P . i}\left(t-k_{I I I i}+1\right) & =\hat{X}_{I I: i}\left(t-k_{I I: i}+1, t+m_{I I: M . P .}\right) \\
= & \hat{D}_{i}\left(t-k_{I I: i}+1, t+m_{I I: M . P .}+l+m_{I I: D . C .}-1\right) .
\end{aligned}
$$

The inventory level of the parts for producing product $i$ at M.P. at the end of day $t$, denoted by $I_{I I: M . P: i}(t)$, is then given by

$$
\begin{aligned}
& I_{I I: M . P: i}(t)=\left[\tilde{I}_{I I: M . P: i}(t)\right]^{+} ; \\
& \tilde{I}_{I I: M . P: i}(t)=I_{I I: M . P: i}(t-1)+Q_{I I: M . P: i}\left(t-k_{I I i}+1\right)-X_{I I: i}(t) .
\end{aligned}
$$

Let $f_{I I}\left(T_{1}, T_{2}\right)$ be the overall profit of Model II generated from the products delivered to the market between day $T_{1}$ and day $T_{2}$. It can be seen that

$$
\begin{aligned}
& f_{I I}\left(T_{1}, T_{2}\right)=\sum_{\tau=T_{1}-1}^{T_{2}-1} \sum_{i=1}^{K}\left\{D_{i}(\tau-1)+\delta\left\{\tilde{I}_{I I: D . C i}(\tau)<0\right\} \cdot \tilde{I}_{I I: D . C i}(\tau)\right\} \cdot\left(P_{i}-T C_{i}-c_{I I D . C . C_{i}}-O P_{I I D . C . C_{i}}\right) \\
& -\sum_{\tau=T_{1}-l}^{T_{2}-l-1} \sum_{i=1}^{K} \frac{I_{I I: M . P .}(\tau-1)+I_{I I: M . P P_{i}}(\tau)}{2} \cdot c_{I I: M . P . i} \cdot r
\end{aligned}
$$

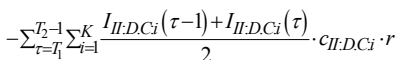

$$
\begin{aligned}
& +\sum_{\tau=T_{1}-1}^{T_{2}-1} \sum_{i=1}^{K} \delta\left\{\tilde{I}_{I I: D C . C_{i}}(\tau)<0\right\} \cdot \tilde{I}_{I: D . C . i}(\tau) \cdot C L_{i},
\end{aligned}
$$

where one has

$$
c_{I I: D . C . i}=c_{I I: M . P . i}+O P_{I I: M . P: i}+I M_{I I I i} .
$$

We first note that $c_{I I: D . C . i}$ in (11) is the unit cost for the final product inventory at D.C. where $c_{I I: M . P: i}$ and $O P_{I I: M . P: i}$ are the procurement cost and the operation cost at M.P. per one unit of product $i$ respectively, and $I M_{I I: i}$ is the import cost per one unit of product $i$. The first term on the right hand side of (10) gives the profit, excluding the inventory costs at M.P. represented by the second term, the inventory costs at D.C. expressed by the third term and the confidence loss costs given in the last term.

\section{Model III: Skeleton Imports}

In Model III, M.P. abroad would produce only Skeletons which are common for all $K$ products. The Skeletons would then be shipped to D.C. in Japan where the final processing for meeting the specifications of the individual products would take place. As before, the confirmed orders of day $t-1$ would be sent to D.C. from the sales offices across Japan through the company's intranet. During day $t$, the ordered products would be processed by incorporating necessary specifications onto Skeletons. By the end of day $t+1$, they would be packaged and shipped out, resulting in the market lead time of three days, provided that the inventory stock is large enough.

In contrast to Model II, Model III requires to estimate only the total demand of the $K$ different products at M.P., which is the major advantage of the SI strategy. More specifically, let

$$
\hat{D}(t)=\sum_{i=1}^{K} \hat{D}_{i}(t) .
$$

It can be seen that

$$
\left.\left.\operatorname{Var} \mid \hat{D}(t)]=\sum_{i=1}^{K} \operatorname{Var} \mid \hat{D}_{i}(t)\right]+2 \sum_{i<j} \operatorname{Cov} \mid \hat{D}_{i}(t), \hat{D}_{j}(t)\right\}
$$

Since the $K$ different products are variations of one product type, they are substitutable in that the demand increase of product $i$ would be likely to result in the demand decrease of other products. Accordingly, one has $\left.\operatorname{Cov} \mid \hat{D}_{i}(t), \hat{D}_{j}(t)\right]<0$ so that $\operatorname{Var}[\hat{D}(t)]<\sum_{i=1}^{K} \operatorname{Var}\left[\hat{D}_{i}(t)\right]$. This means that estimating the total demand can be more accurate than summing the individually estimated demands. Because of this possibility of reducing the estimation error, the safety stock of the Skeletons at D.C. in Model III may be reduced in comparison with that of the final products in Model II. Namely, let the safety stock of the Skeletons at D.C. be to cover $m_{\text {III:D.C. }}$ days worth of the estimated demands. Then, one expects that

$$
m_{I I I: D . C .}<m_{I I: D . C .},
$$

resulting in significant reduction of the inventory cost.

Let $S P_{I I I D . C .}(t)$ be the quantity of Skeletons to be shipped from M.P. to D.C. at the beginning of day $t$. The inventory level of Skeletons at D.C. at the end of day $t$ is denoted by $I_{\text {III:D.C. }}(t)$. We also define $X_{I I I}(t)$ to be the production quantity of Skeletons during day $t$ at M.P. As for Model II, one then sees that

$$
S P_{I I I: D . C .}(t-l+1)=X_{I I I}(t-l)=D\left(t-l, t+m_{I I I: D . C .}-1\right),
$$

and

$$
\begin{aligned}
& I_{\text {III:D.C. }}(t)=\left[\tilde{I}_{I I I: D . C .}(t)\right]^{+} ; \\
& \tilde{I}_{\text {III:D.C. }}(t)=I_{\text {III:D.C. }}(t-1)+S P_{\text {III:D.C. }}(t-l+1)-D(t-1) .
\end{aligned}
$$

Let $k_{I I I: D . C: i}$ be the procurement lead time in days for the parts needed to meet the individual specifications. Let $Q_{\text {III:D.C. } i}(t)$ be the order quantity of such parts for product $i$ placed at the beginning of day $t$. It can be seen that

$$
Q_{I I I: D . C . i}\left(t-k_{I I I: D . C . i}+1\right)=\hat{D}_{i}\left(t-k_{I I I: D . C . i}+1, t+m_{I I I: D . C . i}-1\right) .
$$

The associated inventory levels, denoted by $I_{I I I: D . C . i}(t)$, are then given by

$$
\begin{aligned}
& I_{I I I: D . C . i}(t)=\left[\tilde{I}_{I I I: D . C . i}(t)\right]^{+} ; \\
& \tilde{I}_{I I I: D . C . i}(t)=I_{I I I: D . C . . i}(t-1)+Q_{I I I: M . P .}\left(t-k_{I I I}+1\right)-D_{i}(t-1) .
\end{aligned}
$$

At M.P., the procurement lead time is assumed to be $k_{I I I}$ days, and the safety stock for the parts for producing Skeletons would be to cover $m_{I I I: M . P .}$ days worth of manufacturing operation at M.P. For Skeletons, let 
$Q_{I I I: M . P .}(t)$ and $\hat{X}_{I I I}(\tau, t)$ be defined similarly to $Q_{I I: i}(t)$ and $\hat{X}_{I I: i}(\tau, t)$ respectively. As before, one then sees that

$\hat{X}_{I I I}(\tau, t)=\hat{D}\left(\tau, t+l+m_{I I I: D . C .}-1\right)$.

Accordingly, it follows that

$$
\begin{aligned}
Q_{I I I: M . P .}\left(t-k_{I I I}+1\right) & =\hat{X}_{I I I}\left(t-k_{I I I}+1, t+m_{I I I: M . P .}\right) \\
& =\hat{D}\left(t-k_{I I I}+1, t+m_{I I I: M . P .}+l+m_{I I I: D . C .}-1\right) .
\end{aligned}
$$

The inventory level of the parts for producing Skeletons at M.P. at the end of day $t$, denoted by $I_{I I I: M . P .}(t)$, is then given by

$$
\begin{aligned}
& I_{I I I: M . P .}(t)=\left[\tilde{I}_{I I I: M . P .}(t)\right]^{+} ; \\
& \tilde{I}_{I I I: M . P .}(t)=I_{I I I: M . P .}(t-1)+Q_{I I I: M . P .}\left(t-k_{I I I}+1\right)-X_{I I I}(t) .
\end{aligned}
$$

Let $f_{I I I}\left(T_{1}, T_{2}\right)$ be the overall profit of Model III generated from the products delivered to the market between day $T_{1}$ and day $T_{2}$. The involved costs can be categorized into five classes: the costs directly associated with production and transportation of Skeletons; the inventory cost for keeping the safety stock of Skeletons at D.C.; the inventory costs for keeping the safety stock of parts necessary for product $i$ at D.C.; the inventory cost for keeping the safety stock of parts for producing Skeletons at M.P.; and the cost for losing confidence by failing to meet the demand. It can be seen that

$$
\begin{aligned}
& f_{\text {III }}\left(T_{1}, T_{2}\right) \\
& =\sum_{\tau=T_{1}-2}^{T_{2}-2} \Sigma_{i=1}^{K}\left\{D_{i}(\tau-1)+\delta\left\{\tilde{I}_{\text {IIID.D.C. }}(\tau)<0\right\} \tilde{I}_{\text {IIID.D.C. }}(\tau) \cdot \frac{D_{i}(\tau-1)}{D(\tau-1)}\right\} \cdot\left(P_{i}-T C_{i}-c_{\text {III:D. } i}-c_{\text {III:DC. }}-O P_{\text {III:D.C. } i}\right) \\
& -\sum_{\tau=T_{1}-1}^{T_{2}-2} \frac{I_{\text {III:DC. }}(\tau-1)+I_{\text {III:D.C. }}(\tau)}{2} \cdot c_{\text {III:D.C. }} \cdot r \\
& -\sum_{\tau=T_{1}-1}^{T_{2}-2} \sum_{i=1}^{K} \frac{I_{I I I: D C .}(\tau-1)+I_{I I I D . C . C i}(\tau)}{2} \cdot c_{I I I: D . C . i} \cdot r \\
& -\sum_{\tau=T_{1}-l-1}^{T_{2}-l-2} \frac{I_{I I I M . P .}(\tau-1)+I_{I I I: M . P .} .(\tau)}{2} \cdot c_{I I I M . M . P .} \cdot r \\
& +\sum_{\tau=T_{1}-1}^{T_{2}-2} \Sigma_{i=1}^{K} \delta\left\{\tilde{I}_{\text {III:DC. }}(\tau)<0\right\} \tilde{I}_{\text {IIID.C. }}(\tau) \cdot \frac{D_{i}(\tau-1)}{D(\tau-1)} \cdot C L_{i},
\end{aligned}
$$

where one has

$$
c_{\text {III:D.C. }}=c_{\text {III:M.P. }}+\text { OP III:M.P. }+I M_{\text {III }}
$$

Cost parameters are similar to those for Model II except $c_{\text {III:D.C. } i}$ which describes the cost of parts necessary for producing one unit of product $i$ at D.C.

\section{SIMULATION MODEL}

In year 2005, approximately 1 million laser printers were sold in Japan. The market share of the top maker was about $30 \%$. This means that, on the average, the maker sold 1154 products per day assuming 260 working days a year. Based on this, the daily demands of the total products $D(t) \quad t=1,2, \cdots, 120$ are generated randomly from the distribution of $\mathrm{N}(1200,50)$, where $\mathrm{N}(\mu, \sigma)$ is a normal variate with mean $\mu$ and standard deviation $\sigma$.
These total demands are then allocated into $K$ different products over time using a randomly generated transition probability matrix with the uniform distribution over $[0, K]$ as the initial probability vector. More specifically, let $\underline{P}$ be the randomly generated transition probability matrix and define $\underline{p}^{T}(0)=[1, \cdots, 1] / K$. One then has

$$
\underline{D}^{T}(t)=D(t) \times \underline{p}^{T}(t) ; \underline{p}^{T}(t)=\underline{p}^{T}(t-1) \underline{\underline{P}}, \quad t=1,2, \cdots, 120,
$$

where $\underline{D}^{T}(t)=\left[D_{1}(t), \cdots, D_{K}(t)\right]$. In order to avoid $\underline{p}^{T}(t)$ to approach the ergodic probability vector, the transition probability matrix $\underset{\underline{P}}{=}$ is regenerated every 10 days.

Given $\underline{D}^{T}(t) \quad t=1,2, \cdots, 120$, we next turn our attention to produce the demand estimates $\underline{\hat{D}}^{T}(t) \quad t=1,2, \cdots, 120$. For this purpose, we employ the classical exponential smoothing approach, see e.g. [10]. We recall that $\hat{D}(\tau, t)$ is the total demand of day $t$ estimated at the beginning of day $\tau$. Let $L$ and $\beta$ be the number of terms to be used and the underlying geometric parameter for the exponential smoothing. In order to emphasize the dependence of $\hat{D}(\tau, t)$ on $L$ and $\beta$, we write $\hat{D}(\tau, t \mid \beta, L)$. We begin with $t=\tau$ so that one has

$$
\hat{D}(\tau, \tau \mid \beta, L)=\sum_{j=1}^{L} \theta(j \mid \beta, L) D(\tau-j)+\varepsilon(\beta, L) ; \quad \theta(j \mid \beta, L)=\frac{\beta(1-\beta)^{j-1}}{1-(1-\beta)^{L}} .
$$

Then, the optimal value $\beta^{*}$ is determined so as to minimize $\varepsilon(\beta, L)^{2}$, i.e.

$$
\beta^{*}=\underset{0<\beta<1}{\arg \min }\left[\varepsilon(\beta, L)^{2}\right]
$$

Using this optimal $\beta^{*}$, one then generates

$$
\hat{D}(\tau, \tau)=\hat{D}\left(\tau, \tau \mid \beta^{*}, L\right) ; \quad \hat{D}\left(\tau, \tau \mid \beta^{*}, L\right)=\sum_{j=1}^{L} \theta\left(j \mid \beta^{*}, L\right) D(\tau-j) .
$$

In general, with $t=\tau+m$, one has for $\mathrm{m}=1, \cdots, \mathrm{M}$,

$$
\begin{aligned}
& \hat{D}(\tau, \tau+m)=\hat{D}\left(\tau, \tau+m \mid \beta^{*}, L\right) ; \\
& \hat{D}\left(\tau, \tau+m \mid \beta^{*}, L\right) \\
& =\sum_{j=1}^{m} \theta\left(j \mid \beta^{*}, L\right) \hat{D}(\tau, \tau+m-j)+\sum_{j=m+1}^{M} \theta\left(j \mid \beta^{*}, L\right) D(\tau+m-j) .
\end{aligned}
$$

Simulation for Model III can be carried out through the above procedures. For Model II, Equations (27) and (28) are applied separately to the $K$ individual products. The number of simulation runs is determined in such a way that the relative errors of the cumulative averages of the profit functions in (4), (10) and (22) fall below 0.005 three times consecutively. Parameter values employed for the numerical experiments conducted in this paper are summarized in Table 3.1 on the next page. 
TABLE 3.1

PARAMETER VALUES $\left({ }^{*}: \$ ;{ }^{* *}:\right.$ days $)$

\begin{tabular}{|c|c|c|c|c|c|c|c|}
\hline \multicolumn{2}{|c|}{ Common } & \multicolumn{2}{|c|}{ Model I } & \multicolumn{2}{|c|}{ Model II } & \multicolumn{2}{|c|}{ Model III } \\
\hline$P_{i}$ & 1500 & $k_{I: i}$ & 2 & $\bar{k}_{I I: i}^{*}$ & 2 & $k_{I I I}^{*}$ & 2 \\
\hline$T C_{i}$ & 50 & $m_{I}$ & 3 & $m_{I I: M . P .}$ & 4 & $k_{I I I: D . C: i}$ & 2 \\
\hline$r$ & 0.05 & $c_{I: i}$ & 600 & $m_{I I: D . C .}$ & 3 & $m_{I I I: M . P .}$ & 2 \\
\hline$C L_{i}{ }^{*}$ & 100 & $O P_{I: i}$ & 700 & $c_{I I: M . P: i}$ & 600 & $m_{I I I: D . C .}$ & 2 \\
\hline$K$ & 7 & & & $O P_{I I: M . P: i}$ & 300 & $m_{I I I: D . C . i}$ & 3 \\
\hline$T_{1}$ & 50 & & & $O P_{I I: D . C: i}$ & 50 & $c_{I I I: M . P .}$ & 300 \\
\hline$T_{2}{ }^{* * *}$ & 100 & & & $I M_{I I: i}$ & 100 & $c_{I I I: D . C . i}$ & 300 \\
\hline$L^{* *}$ & 5 & & & & & $O P_{I I I: M . P}$ & 200 \\
\hline$l^{* *}$ & 3 & & & & & $O P_{I I I: D . C: i}$ & 200 \\
\hline & & & & & & $I M_{I I I}$ & 50 \\
\hline
\end{tabular}

\section{DISCUSSION: NUMERICAL RESULTS}

One of the major advantages of the SI strategy in Model III can be found in that estimating the total demand can be more accurate than summing the individually estimated demands in Model II. Because of this, the safety stock at D.C. for Model III can be set smaller than that for Model II as in (14). For the numerical experiments reported in this section, these values are set to be $m_{\text {IIID.C. }}=2$ and $m_{I I D . C .}=3$. Let the demand estimate error for Model II be defined as $\sum_{\tau=T_{1}}^{T_{2}} \sum_{j=1}^{K}\left\{\hat{D}_{j}(\tau)-D_{j}(\tau)\right\}^{2}$, and for Model III, as $\sum_{\tau=T_{1}}^{T_{2}}\{\hat{D}(\tau)-D(\tau)\}^{2}$. These values are summarized in Table 4.1. It can be seen that the demand estimate error for Model III is approximately three times smaller than that for Model II at D.C. This ratio exceeds four at M.P., demonstrating the validity of the SI strategy.

In Fig. 4.1, the overall profit functions are exhibited as a function of the oversea operation costs at M.P. while the domestic operation costs at D.C. are fixed. One can see that when the cost difference between D.C. and M.P. is small, the complete oversea production in Model II exceeds the SI strategy in Model III. As the difference becomes larger, however, Model III supersedes Model II. In light of the trend that the labor costs in developing countries have been steadily increasing, this fact justifies the adoption of the SI strategy by Japanese manufacturing corporations.

\section{CONCLUSION}

This paper develops simulation models for analyzing the positive effects of the SI strategy, which is a new phenomenon recently practiced by certain Japanese manufacturing corporations. Numerical results reveal that the SI strategy is justifiable when the oversea operation costs exceed a certain level in comparison with the domestic operation costs.
TABLE 4.1

DEMAND ESTIMATE ERROR

\begin{tabular}{|r|r|r|}
\hline & At D.C. & At M.P. \\
\hline Model II & 1,087 & 1,518 \\
\hline Model III & 367 & 374 \\
\hline
\end{tabular}

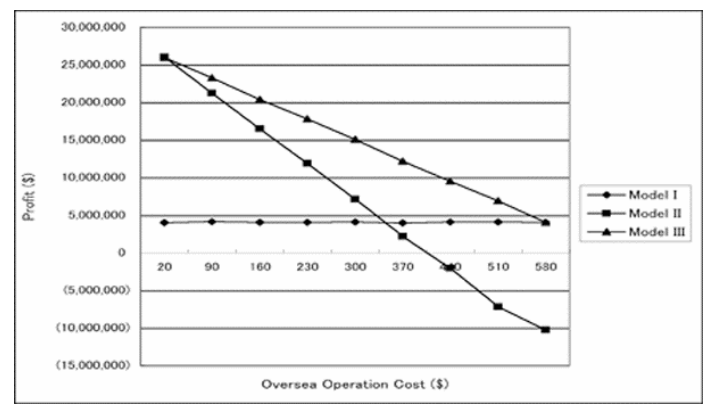

Fig. 4.1. Performance comparison of three models.

\section{ACKNOWLEDGMENT}

This research is supported by MEXT Grant-in-Aid for Scientific Research (C) 17510114.

\section{REFERENCES}

[1] E. Helpman, M. J. Melitz, and S. R. Yeaple, "Export Versus FDI," NBER Working Paper, No.9439, 2003.

[2] E. Helpman, M. J. Melitz, and S. R. Yeaple, "Export Versus FDI with Heterogenous Firms," American Economic Review, vol.94, pp. 300-316, March 2004.

[3] T. M. Greaney, "Reverse Importing and Asymmetric Trade and FDI: A Networks Explanation," Journal of International Economics, vol.61, pp. 453-465, 2003.

[4] R. Rob and V. Nikolaos, "Foreign Direct Investment and Exports with Growing Demand," Review of Economic Studies, vol.70, pp. 629-648, July 2003.

[5] I. J. Horstmann and J. R. Markusen, "Endogenous Market Structures in International Trade (natura facit saltum)," Journal of International Economics, vol.32, pp. 109-129, 1992.

[6] M. Yomogida, "Fragmentation, Welfare, and Imperfect Competition," COE/RES Discussion Paper Series, No.102, Hitotsubashi University, 2004.

[7] K. Matsubara, "FDI with Reverse Imports and Hollowing Out," presented at Fall 2004 Midwest International Economics and Economic Theory Meeting, Washington University in St. Louis, U.S.A., November 2004.

[8] K. Cowling and P. R. Tomlinson, "The Japanese Crisis - A Case of Strategic Failure?” Economic Journal, vol.110, pp. 358 -381 , June 2000.

[9] N. Gaston and D. Nelson, "Structural Change and the Labormarket Effects of Globalization," Review of International Economics, vol.12, pp. 769-792, November 2004.

[10] R. G. Brown, Statistical Forecasting For Inventory Control, New York: McGraw-Hill, 1959. 